\title{
Huygens Subgridding for 3D Frequency-Dependent Finite-Difference Time-Domain Method
}

\author{
Maksims Abalsenkovs, Fumie Costen, Jean-Pierre Bérenger, Ryutaro Himeno, Hideo Yokota and Masafumi Fujii
}

\begin{abstract}
Subgridding methods are often used to increase the efficiency of the wave propagation simulation with the Finite-Difference Time-Domain method. However, the majority of contemporary subgridding techniques have two important drawbacks: the difficulty in accommodating dispersive media and the inability for physical interfaces to cross the subgridding interface.

This work presents an extension of the frequency dependent Huygens Subgridding method from one dimension to three dimensions. Frequency dependency is implemented via the Auxiliary Differential Equation approach using the one-pole Debye relaxation model. Numerical experiments indicate that subgridding interfaces can be placed in various Debye media as well as across the physical interface.
\end{abstract}

Index Terms-Computational electromagnetics, Electromagnetic fields, Electromagnetic modeling, Finite difference methods, Multigrid methods, Numerical simulation

\section{INTRODUCTION}

$\mathbf{M}$ ODERN engineering problems grow in size and at the same time require precise knowledge about electromagnetic behaviour within a fine geometry. Prior to prototyping, electromagnetic field distribution is identified using numerical simulation techniques such as the Finite-Difference TimeDomain (FDTD) method. A precise FDTD simulation requires a high spatial resolution. Wavelengths of interest must be sampled by at least 10 grids, while the time step must be small enough to satisfy the Courant-Friedrichs-Lewy (CFL) stability criterion [1]. Therefore the FDTD solution of a realistic problem is usually computationally expensive.

Subgridding techniques can significantly reduce the computational burden and enlarge the application domain of the FDTD method. This is the purpose of the present paper, devoted to the extension of the Huygens subgridding method to frequency dependent media.

Inclusion of material dispersion into FDTD simulations is of growing importance. In broadband systems the dielectric parameters of a medium are functions of frequency [2]. Authorised,

Manuscript received April 12, 2012; revised April 12, 2012.

M. Abalenkovs is with EM Software \& Systems GmbH, Otto-LilienthalStrasse 36, 71034, Böblingen, Germany, e-mail: m.abalenkovs@emss.de

F. Costen is with the School of Electrical and Electronic Engineering, The University of Manchester, Manchester, M13 9PL, United Kingdom, fumie. costen@manchester.ac.uk

J.-P. Bérenger is with the Centre d'Analyse de Défense, 16 bis, Avenue Prieur de la Côte d'Or, 94114, Arcueil, France, e-mail: berenger@ieee.org

R. Himeno is VCAD System Research Program, RIKEN,Wako, Saitama,351-0198, Japan, himeno@ riken.jp

H. Yokota is with the B10-Research Infrastructure Construction Team, Advanced Technology Support Division,Advanced Science Institute, RIKEN, Saitama, Japan, hyokota@ riken.jp

M. Fujii is with the Faculty of Engineering, University of Toyama, Japan, mfujii@eng.u-toyama.ac.jp license-free use of the Ultra-Wideband (UWB) signal [3] has stimulated a rapid development of UWB applications such as biomedical and through-wall imaging, positioning systems, ground penetrating radars and remote monitoring.

Subgridding methods increase computational efficiency by decomposing the simulation domain into several separate domains with different temporal and spatial increments. Subgridding allows the system to obey the CFL condition in each grid independently. Subgridding techniques may be divided into two major groups: usual and hybrid subgridding. In usual subgridding the simulation domain is composed of FDTD volumes with different spatio-temporal increments. Subgridding interface acts as a boundary condition of both grids, where main grid components are interpolated and subgrid components extrapolated prior to conventional FDTD updates. Hybrid subgridding uses a combination of the FDTD method and the Finite Element Method (FEM). The FDTD method discretises the main grid, while the entire subgrid or only an interface region is constructed with Finite Elements (FEs). In hybrid subgridding small spatial steps can be used with large temporal steps in the FE region. Application of large temporal steps throughout the main and subgrids allows avoidance of temporal interpolation.

Stability and material traversal are two weak points of most modern subgridding algorithms. Due to different numerical dispersion in the main- and sub-grids, and imperfect spatial interpolation at the interface, many subgridding methods suffer from instabilities. Material traversing subgridding [4]-[6] enables dielectric media to cross the subgrid and especially the subgridding interface. Very few subgridding algorithms allow material traversal because materials crossing the subgridding interface may create additional interface reflection and instability. Collocated subgridding can be used [4], where only magnetic field components have to be interpolated on the interface. The main grid and the sub grid share the electric field. In this way collocated subgridding allows non-magnetic material crossing. Subgridding with domain overriding [5] use the overlapping region to eliminate and enforce the dielectric material parameters traversing the interface. Material traversal can be enabled by extending the subgridding approach to Lorentz-Drude material model [6].

Human body applications of subgridding methods remain scarce. Subgridding with the highest ratio of 7 was used for the dosimetry in the inner ear [7].

[8] studied electromagnetic wave propagation in the interstitial applicators used in bone cancer treatment.

[9] simulated current distribution inside the human torso expressing dispersive materials with reluctivity, electric con- 
ductivity and permittivity.

Huygens Subgridding (HSG) is one of relatively new and promising subgridding methods $[10]-[12]$ in the category of usual subgridding. [13] has proposed Huygens Subgridding for the Frequency-Dependent Finite-Difference Time-Domain (HSG-FD-FDTD) method in one dimension (1D) and applied it with the subgridding ratio of 15 to a wave propagation problem in the human torso. However, practical electromagnetic problems are three-dimensional (3D) and can not be properly represented in a $1 \mathrm{D}$ simulation.

Objectives of this work are: (i) extension of the HSGFD-FDTD [13] method from 1D to 3D and (ii) application of the 3D HSG-FD-FDTD algorithm to calculate the wave propagation inside the human torso.

\section{FREQUENCY-DEPENDENT-}

\section{FINITE-DIFFERENCE TIME-DOMAIN METHOD}

Based on Taylor series expansion, the Frequency-Dependent Finite-Difference Time-Domain (FD-FDTD) algorithm approximates the derivatives of Maxwell's equations with the central difference operations. Electric flux density is derived from the source-free Ampère's law:

$$
\frac{\partial \boldsymbol{D}}{\partial t}=\nabla \times \boldsymbol{H}
$$

where $\boldsymbol{D}$ and $\boldsymbol{H}$ denote the electric flux density and the magnetic field, while $t$ is time.

The electric field is obtained from the constitutive expression for the electric flux density. This intermediate calculation step is called the Auxiliary Differential Equation (ADE) approach and allows incorporation of frequency dependency into the original FDTD method:

$$
\boldsymbol{D}=\varepsilon \boldsymbol{E}=\varepsilon_{0} \varepsilon_{r} \boldsymbol{E}=\varepsilon_{0}\left(\varepsilon_{\infty}+\frac{\sigma}{\jmath \omega \varepsilon_{0}}+\frac{\varepsilon_{s}-\varepsilon_{\infty}}{1+\jmath \omega \tau}\right) \boldsymbol{E},
$$

where $\varepsilon, \varepsilon_{r}, \varepsilon_{0}, \varepsilon_{s}, \varepsilon_{\infty}$ are the electric permittivities: generic, relative, vacuum, relative static and relative optical. Symbols $\boldsymbol{E}, \sigma, \jmath, \omega, \tau$ stand for the electric field, electric conductivity, imaginary unit, angular frequency and the relaxation time, respectively. The expression in parentheses in (2) is the one-pole Debye relaxation model which presents the relative electric permittivity as a frequency-dependent complex number.

After multiplication and grouping the $(\jmath \omega)$ and $(\jmath \omega)^{2}$ terms (2) becomes:

$(\jmath \omega)^{2} \tau \boldsymbol{D}+\jmath \omega \boldsymbol{D}=(\jmath \omega)^{2} \tau \varepsilon_{0} \varepsilon_{\infty} \boldsymbol{E}+\jmath \omega\left(\sigma \tau+\varepsilon_{0} \varepsilon_{s}\right) \boldsymbol{E}+\sigma \boldsymbol{E}$.

Taking into account the time dependence of $\exp (\jmath \omega t)$ transforms into:

$\frac{\partial^{2}(\tau \boldsymbol{D})}{\partial t^{2}}+\frac{\partial \boldsymbol{D}}{\partial t}=\frac{\partial^{2}\left(\tau \varepsilon_{0} \varepsilon_{\infty} \boldsymbol{E}\right)}{\partial t^{2}}+\frac{\partial\left(\left(\sigma \tau+\varepsilon_{0} \varepsilon_{\infty}\right) \boldsymbol{E}\right)}{\partial t}+\sigma \boldsymbol{E}$.

Approximating the time derivatives with finite differences and expressing $\boldsymbol{E}$ from (4) yields the final form of the ADE used in the FD-FDTD method.

$\boldsymbol{H}$ is obtained from the source-free Faraday's law and the constitutive expression,

$$
\frac{\partial \boldsymbol{B}}{\partial t}=-\nabla \times \boldsymbol{E}, \quad \boldsymbol{B}=\mu \boldsymbol{H} \quad \Rightarrow \quad \frac{\partial \boldsymbol{H}}{\partial t}=-\frac{1}{\mu} \nabla \times \boldsymbol{E},
$$

where $\boldsymbol{B}$ and $\mu$ denote the magnetic flux density and magnetic permeability.

In summary, the FD-FDTD method solves the discretised versions of (1), (4) and (5) on a spatial grid in time domain following the calculation order of $\boldsymbol{D} \rightarrow \boldsymbol{E} \rightarrow \boldsymbol{H}$.

\section{HUYGENS SUBGRIDDING PRINCIPLES}

HSG has multiple advantages over conventional subgridding algorithms. Instead of direct connection between the main and the subgrids, HSG operates by means of equivalent currents flowing through Huygens Surfaces (HSs). In contrast to normal subgridding techniques, which suffer from spurious interface reflection due to an abrupt change of the mesh size, application of HSs dramatically reduces the spurious interface reflection and removes the need for field extrapolation at the interfaces [12].

In HSG the subgridding ratio is defined as $r=\Delta s_{a} / \Delta s_{b}=$ $\Delta t_{a} / \Delta t_{b}$, where $\Delta s, \Delta t$ denote the spatial and temporal steps, and indices $a, b$ stand for the main and the subgrid. Distinct temporal steps $\Delta t_{a}, \Delta t_{b}$ allow each calculation domain to maintain its own stability condition. The only serious drawback of HSG is the instability. However, the introduction of low-pass filters $[10]$ to the process at the interface between the main grid and the sub grid can make HSG stable.

The core idea of HSG is the presentation of the original problem domain as a collection of artificial equivalent domains. HSG decomposes the physical problem into a main domain and one or several subdomains.

Recall the equivalence theorem. Consider a spatial domain which consists of two parts separated by a surface. The equivalence theorem states that an electromagnetic field generated by the sources in one part of the domain can be reproduced in the other part of the domain by impressing the electric and magnetic currents $\boldsymbol{J}_{s}, \boldsymbol{M}_{s}$ onto the separating surface:

$$
\boldsymbol{J}_{s}=\boldsymbol{n} \times \boldsymbol{H}_{i}, \quad \boldsymbol{M}_{s}=-\boldsymbol{n} \times \boldsymbol{E}_{i},
$$

where $\boldsymbol{E}_{i}, \boldsymbol{H}_{i}$ are the electric and magnetic fields which would exist on the separating surface, if the sources were present and $\boldsymbol{n}$ was a unit vector normal to the surface, directed opposite to the sources. Surface upon which the equivalent currents are flowing is called a Huygens Surface.

Inner Huygens Surface (IS) and Outer Huygens Surface (OS) transfer electromagnetic signals between the main and the subgrids. Fig. 1 shows the OS and IS individually and combined. For simplicity, in Fig. 1 the OS and IS are represented as spheres. Both the OS and IS can be viewed as two independent spheres of the equivalence theorem. The OS sphere radiates the electromagnetic fields from inside out and the IS sphere from outside in. The OS and IS spheres combined implement the OS-IS interface, which passes electromagnetic fields between the main and the subgrid domains. See [10] for a detailed explanation of HSs.

\section{A. Inner surface equations}

At each subgrid FDTD iteration, after the regular update of the electric flux density $D_{b}$, an equivalent current $M_{s}$ is 

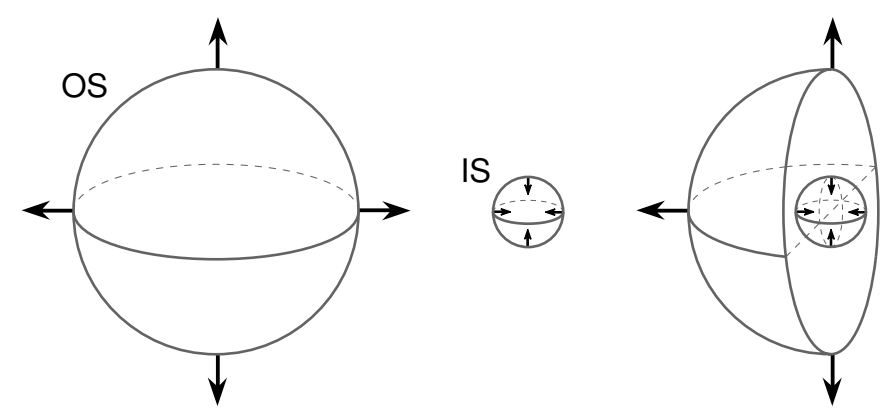

Fig. 1. HSG principles. The leftmost and the central spheres represent the Outer and the Inner Huygens Surfaces (OS, IS) depicted separately. The rightmost hemisphere shows the IS inside of the OS. Outwards directed arrows show the equivalent current flowing from the sub- to the main grid. Inwards pointing arrows depict the equivalent current flowing from the main to the subgrid.

injected onto the $\boldsymbol{D}_{\boldsymbol{b}}$ values at IS. $\boldsymbol{H}_{\boldsymbol{a}}$ influences $\boldsymbol{D}_{\boldsymbol{b}}$ by means of [14];

$$
D_{z b(\text { (IS node })}^{t_{b}+\Delta t_{b}}=D_{z b(\text { IS node })}^{t_{b}} \pm \frac{\Delta t_{b}}{\Delta x_{b}} \widetilde{H}_{y a(\text { IS node })}^{t_{b}+\Delta t_{b} / 2}
$$

where IS node lies in the FDTD cell located closest to the IS. Tilde on top of $H_{y a}$ denotes the temporal interpolation described in $[10]-[12]$. The sign preceding $\widetilde{H}_{y a(\text { IS node })}$ depends on the spatial location of the given component relative to the IS and is governed by the Total-Field/Scattered-Field (TF/SF) approach [14]. (7) only applies to an IS perpendicular to $x$ direction. Similar equations are used for IS perpendicular to $y$ and $z$ axes, which update $\boldsymbol{D}_{\boldsymbol{x} \boldsymbol{b}}$ and $\boldsymbol{D}_{\boldsymbol{y} \boldsymbol{b}}$.

$\boldsymbol{E}_{\boldsymbol{b}}$ at the IS is calculated immediately after $\boldsymbol{D}_{\boldsymbol{b}}$ is updated.

The influence equation for $\boldsymbol{H}_{b}$ at the IS remains unchanged in comparison to the frequency-independent HSG [10]-[12]. For example, (8) shows the $E_{y a}$ influence on the $H_{z b}$ on the IS normal to $x$ :

$$
H_{z b(\text { IS node })}^{t_{b}+3 \Delta t_{b} / 2}=H_{z b(\text { IS node })}^{t_{b}+\Delta t_{b} / 2} \pm \frac{\Delta t_{b}}{\mu_{0} \Delta x_{b}} \widetilde{E}_{y a(\text { IS node })}^{t_{b}+\Delta t_{b}} .
$$

\section{B. Outer surface equations}

Since the subgrid values overlap with the main grid points in space and time, no spatial or temporal extrapolations are required in the OS influence, which remain unchanged relative to the frequency-independent HSG [10]-[12]. For example, $\boldsymbol{D}_{\boldsymbol{z} \boldsymbol{a}}$ and $\boldsymbol{H}_{\boldsymbol{z} \boldsymbol{a}}$ on the OS perpendicular to $x$ are advanced,

$$
\begin{aligned}
& D_{z a(\text { OS node })}^{t_{a}+\Delta t_{a}}=D_{z a(\text { OS node })}^{t_{a}} \pm \frac{\Delta t_{a}}{\Delta x_{a}} H_{y b(\text { OS node })}^{t_{a}+\Delta t_{a} / 2}, \\
& H_{z a(\text { OS node })}^{t_{a}+3 \Delta t_{a} / 2}=H_{z a(\text { OS node })}^{t_{a}+\Delta t_{a} / 2} \pm \frac{\Delta t_{a}}{\mu_{0} \Delta x_{a}} E_{y b(\text { OS node })}^{t_{a}+\Delta t_{a}},
\end{aligned}
$$

where the OS node lies in the FDTD cell located closest to the OS. Equations for the field components in the $x$ and $y$ directions are obtained in a similar manner. $E_{z a}$ values are calculated using (4) immediately after the $D_{z a}$ values were updated in 9 .
TABLE I

MEdia PARAMETERS FOR THE ONE-POLE DEB YE MODEL

\begin{tabular}{lrrrr}
\hline Medium & $\boldsymbol{\sigma}[\mathrm{S} / \mathrm{m}]$ & $\varepsilon_{s}$ & $\varepsilon_{\infty}$ & $\boldsymbol{\tau}[\mathrm{ps}]$ \\
\hline Air & 0.0000 & 1.0000 & 1.0000 & 0.0000 \\
Fat & 0.0371 & 5.5307 & 3.9981 & 0.2363 \\
Heart & 1.0188 & 63.5490 & 34.9097 & 0.2886 \\
Bone & 0.1040 & 14.1691 & 7.3633 & 0.3411 \\
Muscle & 0.7471 & 56.9315 & 28.0013 & 0.1867 \\
\hline
\end{tabular}

\section{NUMERICAL RESUlts}

A variety of testing scenarios were designed to verify HSG performance in dispersive media. The stability and accuracy of the HSG were examined in artificial settings as well as in a realistic scenario. Also the computational efficiency of the HSG with $r=5$ was compared to an all fine-grid FDTD method.

\section{A. Simulation settings for basic accuracy tests}

All tests were launched with a subgridding ratio $r$ of 5 . No low-pass filtering was used in the HSG. A Perfectly Matched Layer (PML) of 10 and 6 cells bounded the simulation domains in the main and the subgrid regions, respectively. The main and the subgrids without the PML were comprised of $60^{3}$ and $121^{3}$ grid points. The volume within the Inner Surface occupied $16^{3}$ cells of the main grid. Simulations were performed for 2000 time steps unless stated otherwise. Spatial and temporal steps were $\Delta s_{a}=10 \mathrm{~mm}$ and $\Delta s_{b}=2 \mathrm{~mm}$; and $\Delta t_{a}=17.91 \mathrm{ps}$ and $\Delta t_{b}=3.58 \mathrm{ps}$.

In this work the HSG and the FDTD signify the frequencydependent HSG-FDTD and the frequency-dependent FDTD methods, respectively. The main and the subgrid denote the main and the subgrid regions of the HSG, while the words "coarse" and "fine" grids refer to the FDTD methods comprised of all coarse and all fine cells.

Highest frequency of interest $f_{\max }$ is $6 \mathrm{GHz}$, given a $-20 \mathrm{~dB}$ threshold value of the frequency spectrum of the excitation. Calculated as $\chi=c /\left(f_{\max } \Delta s\right)$, the spatial resolution of the main and the coarse grids corresponds to 5, and the suband the fine grids to 25 elements per wavelength. Symbol $c$ in the above expression denotes the speed of light propagation in vacuum.

$z$-directed soft point source [2] was used for excitation. An excitation waveform is defined by means of the following Gaussian pulse: $\exp \left(-\left(2 t f_{\max }-3\right)^{2}\right)$. In all experiments reported in Section IV $f_{\max }$ equals $6 \mathrm{GHz}$.

Media parameters for the Debye model used in the basic scenarios are listed in Table II The media parameters for all human tissues, which are used in Section IV-D are presented in [15].

In this work the Air, Fat and Bone media are called the weak Debye media as their conductivity is lower than the conductivity of the Heart or Muscle. The Heart and Muscle tissues are referred to as the strong Debye media.

\section{B. Basic accuracy tests}

Fig. 2 2 shows a generic setting for the basic accuracy tests of Scenarios 1, 2 and 3. Scenario settings of these three cases are 


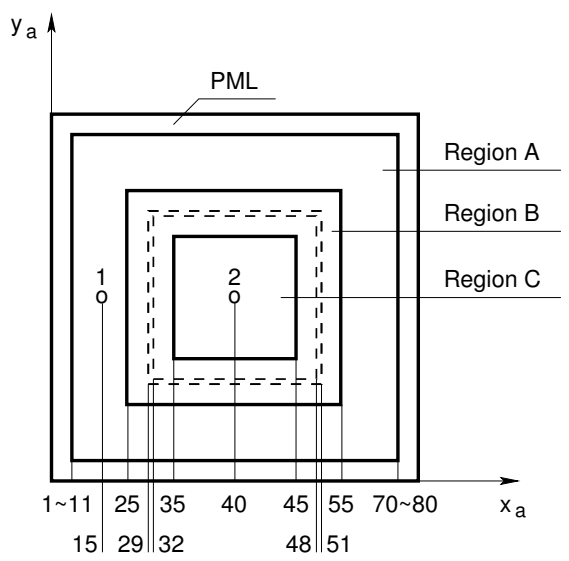

Fig. 2. Cross-section of Scenario 1, 2 and 3 at the plane $z_{a}=40$. Dotted lines denote the Outer and Inner Huygens Surfaces (OS, IS). Symbol "o" marks the locations of interest at points 1 and 2. Scenarios 1,2 and 3 are symmetric relative to the centre line. Cross-section at the plane $y_{a}=40$ is the same as presented here.

TABLE II

RADIO ENVIRONMENT SETTINGS OF SCENARIOS 1, 2 AND 3

\begin{tabular}{cccccc}
\hline $\begin{array}{c}\text { Scenario } \\
\text { number }\end{array}$ & $\begin{array}{c}\text { Region } \\
\text { A }\end{array}$ & $\begin{array}{c}\text { Region } \\
\text { B }\end{array}$ & $\begin{array}{c}\text { Region } \\
\text { C }\end{array}$ & $\begin{array}{c}\text { Excitation } \\
\text { location }\end{array}$ & $\begin{array}{c}\text { Observation } \\
\text { location }\end{array}$ \\
\hline 1 & Air & Air & Heart & P1 & P2 \\
2 & Air & Fat & Heart & P1 & P2 \\
3 & Air & Fat & Heart & P2 & P1 \\
\hline
\end{tabular}

presented in Table II Symbols P1 and P2 in Table II denote the points 1 and 2 in Fig. 2. The cartesian coordinates of the points 1 and 2 are $(15,40,40)$ and $(40,40,40)$ respectively.

1) HSG in the Air with the main grid excitation: Scenario 1 is effectively the same as in Fig. 9 in [12] in that the IS and OS are placed in the free space. Fig. 3 presents $E_{z}$ obtained with the HSG, the FDTD on the all coarse grid and the FDTD on the all fine grid. The mesh sizes of the coarse and fine grids are the same as those of the main and subgrids of the HSG.

The HSG signal matches the fine grid signal very well, because the Heart is in the subgrid of the HSG. Conversely, the highest frequencies of the coarse grid signal cannot properly propagate in the Heart, which resides in the coarse grid. Therefore the coarse grid simulation produces an erroneous signal, seen in Fig. 3 Good matching between the HSG and the fine grid signals confirms that the HSG behaves the same way as in [12].

2) HSG in the homogeneous dispersive medium with the main grid excitation: Scenario 2 Table II illustrates the HSG performance when the OS-IS interface is placed in a dispersive medium. The signal travels through Air towards the subgrid region and first enters a weak Debye medium and then a strong Debye medium.

Results in frequency domain are plotted in Fig. 4 The fine and the coarse grid results are substantially different. This is because the highest frequencies of the signal cannot be sampled properly by the coarse mesh in the Heart medium. Conversely a good matching is observed between the HSG signal and the fine grid signal, because the Heart is within the subgrid of the HSG calculation.

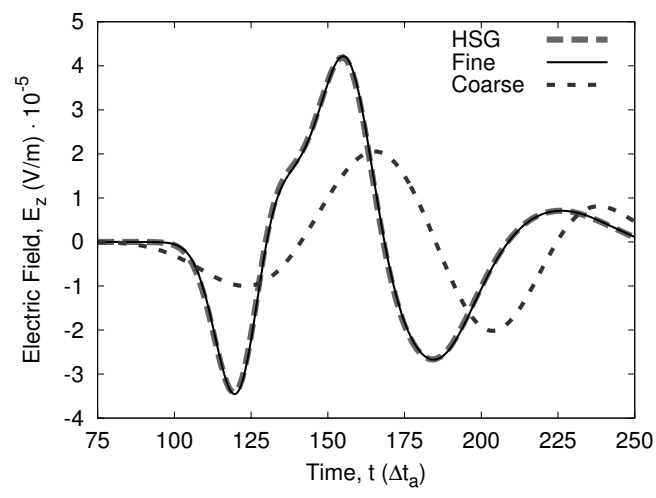

Fig. 3. Time domain observation at P2 in Scenario 1. "Coarse" and "Fine" denote the signals obtained with the FDTD method on the all coarse and the all fine grids. Instability in the HSG is observed at approximately $1000 \Delta t_{a}$.

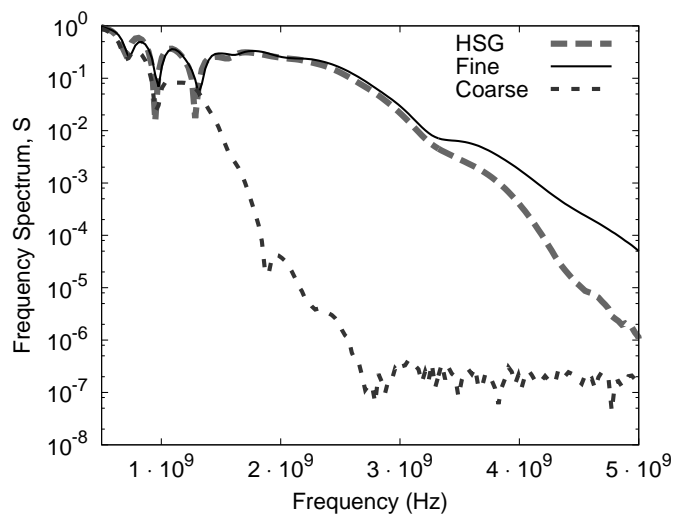

Fig. 4. Frequency domain observation at P2 in Scenario 2. In time domain no instability is visible during the entire simulation of 2000 time steps.

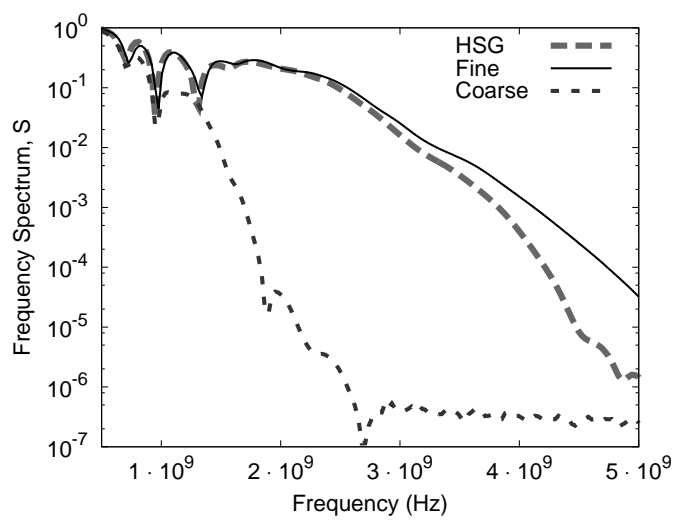

Fig. 5. Frequency domain observation at P1 in Scenario 3. In time domain no instability is visible during the entire simulation time of 2000 time steps.

The difference at the highest frequencies is explained by the wave propagation in the Fat medium, which lies in the main grid of the HSG, outside the OS. Fig. 4 shows the advantage of using the HSG. Applying the fine grid only in the subgrid region of the HSG allows to be obtained a result comparable to the one achieved using a fine grid in the entire computational domain. 


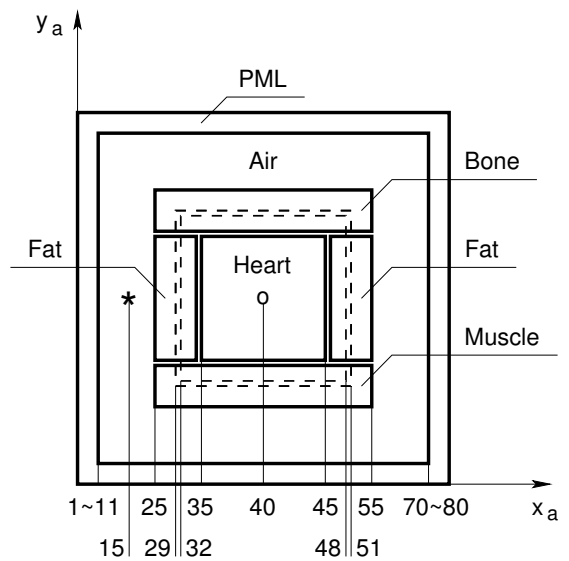

Fig. 6. Cross-section of Scenario 4 at the plane $z_{a}=40$. The OS-IS interface is placed in the Fat, Bone and Muscle media. Symbols "*" and "o" denote the excitation and the observation locations, respectively.

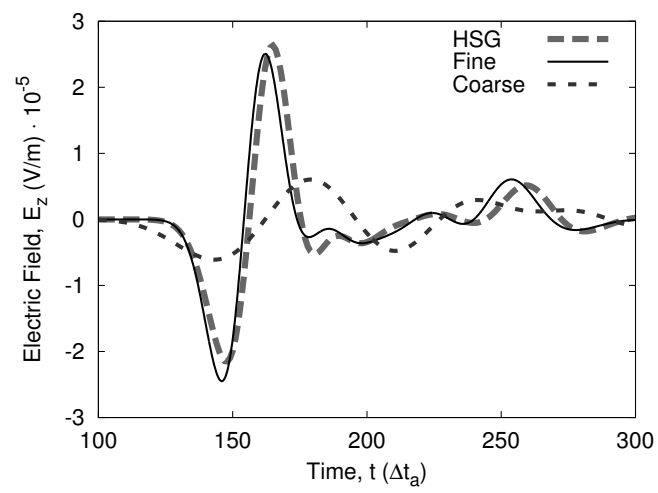

Fig. 7. Time domain observation of Scenario 4. Close matching between the HSG and the fine grid signals is observed. A small drift of the HSG from the fine grid signal is explained by the initial wave propagation in the main grid inside the Air and Fat media. No instability is visible over the entire simulation time.

3) HSG in the homogeneous dispersive medium with the subgrid excitation: Scenario 3 is the opposite of Scenario 2 with the excitation source placed at P2 in the Heart and the observation at P1 in the Air. Fig. 5 shows the observation results. As expected, the signals in Fig. 5 resemble the signals in Fig. 4. The good matching of the HSG signal with the fine grid signal is preserved.

4) HSG in the inhomogeneous dispersive media with main grid excitation: Scenario 4, shown in Fig. 6, offers a more realistic setting of the radio environment. Four dispersive media compose the subgrid. The Fat, Bone and Muscle tissues cross the HSs, while the Heart occupies the entire subgrid. This test supports the correct HSG behaviour when multiple different media intersect the HSs. Fig. 7 shows a good matching between the HSG and the fine grid signals.

\section{Stability}

The HSG method in the Air is unstable. The instability becomes visible after several thousands of time steps [12]. Based on the previous experimental results from the Scenarios 1 to 4 and [13] a hypothesis arises that dispersive media contribute

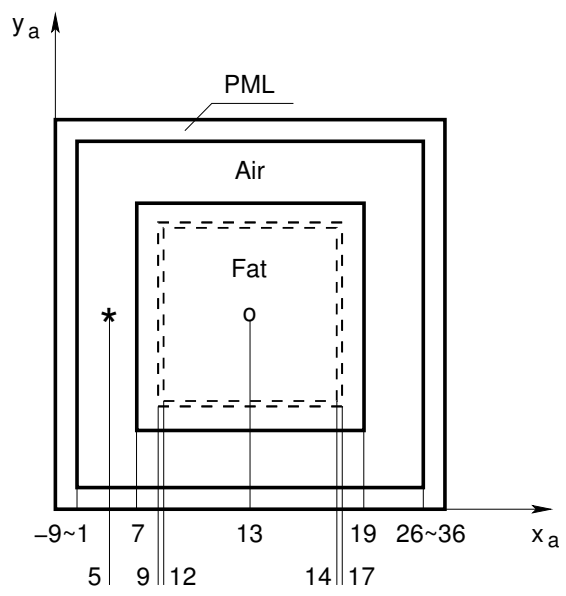

Fig. 8. Cross-section of Scenario 5 at the plane $z_{a}=13$. Scenario 5 is symmetric relative to the centre line. Cross-section at the plane $y_{a}=13$ is the same as presented here.

to a better HSG stability. A strong dispersive medium delays the visibility of the native HSG instability.

Scenario 5 tests the effect of $\sigma$ of the Debye medium on the overall stability of the dispersive HSG simulation. The setting of Scenario 5 is depicted in Fig. 8. Domain sizes of $46^{3}$ and $63^{3}$ were applied to the main and the subgrids. The simulation was run for $10^{6} \Delta t_{a}$. The spatial and temporal steps were $\Delta s_{a}=0.9 \mathrm{~mm}, \Delta s_{b}=0.18 \mathrm{~mm}$ and $\Delta t_{a}=1.612 \mathrm{ps}, \Delta t_{b}=0.322 \mathrm{ps}$.

The Fat tissue was taken for a basis medium (See Table I] and [15] for the exact values of the media parameters). The experiments were performed with a modified $\sigma$ of Fat given by $\sigma=0[\mathrm{~S} / \mathrm{m}]$ and $\sigma=3.71 \cdot 10^{2 m},[\mathrm{~S} / \mathrm{m}]$ where $m$ was an integer veried from -1 to 1 . This constituted four test cases with the constant values of $\varepsilon_{s}, \varepsilon_{\infty}, \tau$ and the changing value of $\sigma$. Only the case when $m=-1$ corresponded to the real Fat tissue, while the other three cases resulted in the wave propagation in an artificial medium.

The results shown in Fig. 9 support the aforementioned hypothesis-no instability growth was observed in the HSG when a non-zero electric conductivity is applied. Most likely minor amplitude fluctuations after the main peaks (at time $t \geq 8 \cdot 10^{3} \Delta t_{a}$ ) are attributed to numerical noise. The case when $\sigma=0$ diverged after approximately $1.7 \cdot 10^{5} \Delta t_{a}$.

\section{Accuracy in the realistic scenario}

1) Defibrillation simulation: Current Resuscitation Guidelines [16] pose the need for in-depth research on defibrillators, aimed at increasing the defibrillation success rate. Successful defibrillation depends on many factors: current level, defibrillator waveform, electrode size, shape and position, and transthoracic impedance. Practical tests of defibrillators are difficult and inhumane. On the other hand an efficient electromagnetic wave propagation solver becomes a versatile tool for defibrillator optimisation. The HSG method can be applied to simulate electromagnetic waves propagating from the defibrillator pads through the human body. Multiple defibrillator parameters can be optimised with a numerical simulation of the wave propagation in a digital human phantom. The HSG 


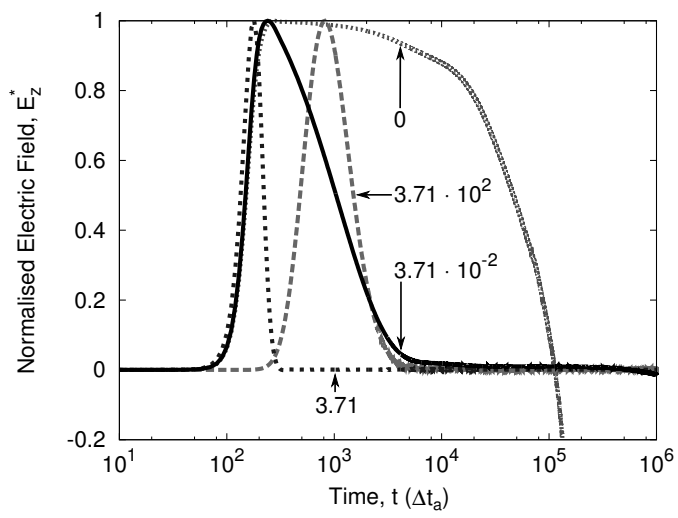

Fig. 9. Time domain observation of Scenario 5. The cases when $\sigma=0$ and $\sigma=3.71 \cdot 10^{n}$, where $n$ is $-2,0$ and 2 are plotted. In case of $\sigma=0$ the signal diverges after approximately 170000 time steps.

will provide a high mesh resolution throughout the heart and a low throughout the torso, resulting in the sufficiently accurate solution.

2) Simulation settings: The digital human phantom (DHP) used in this scenario was provided by RIKEN (Saitama, Japan). The usage was approved by RIKEN ethical committee. The original spatial resolution of the DHP was $1 \mathrm{~mm}$. The phantom consisted of 53 distinct tissues. For the HSG computations the main grid size was $81 \times 106 \times 182$ cells and the subgrid size was $182 \times 193 \times 171$ cells. These sizes include the four and six points thick PML terminating the main and the subgrids respectively. The torso lay entirely within the main grid, with several cells of Air in-between the torso and the surrounding PML. In the subgrid, the Heart lay entirely within the IS. Ranges of the actual FDTD domains were $\left\{x_{a}, y_{a}, z_{a}\right\} \in\{[-13,67],[-3,102],[160,341]\}$ in the main grid and $\left\{x_{b}, y_{b}, z_{b}\right\} \in\{[90,271],[115,307],[1138,1308]\}$ in the subgrid.

The spatial steps were $\Delta s_{a}=5 \mathrm{~mm}, \Delta s_{b}=1 \mathrm{~mm}$, and the temporal were $\Delta t_{a}=8.955 \mathrm{ps}, \Delta t_{b}=1.791 \mathrm{ps}$. For comparison the wave propagation inside the human torso was also simulated with the FDTD method using the all coarse and all fine grids.

Two defibrillator pads were placed on the torso, one at the front and one at the rear as illustrated in Fig. 10. The size of each defibrillator pad equalled to $7.5 \times 10 \mathrm{~cm}$. Scenario setting on the cross-section line $x_{a} \in[-13,67], y_{a}=42, z_{a}=244$ is presented in Fig. 11, where Fig. 11a gives a general scenario overview, Fig. $11 \mathrm{~b}$ shows the all coarse grid and Fig. 11c depicts the all fine grid views of the setting.

Points on each pad were excited in-phase, while both pads were excited anti-phase. The excitation waveform was a Gaussian pulse defined in Section IV-A The simulation was performed for $10^{3} \Delta t_{a}$.

The Skin was situated in the main grid. The width of the Skin in the human phantom constituted $1 \mathrm{~mm}$, which corresponded to one $\Delta s_{b}$ or one fifth of $\Delta s_{a}$. Therefore, Debye parameters of the Skin medium for the main grid of the HSG were divided by 5 . This would be rigorous if the constitutive parameters of the medium were independent of

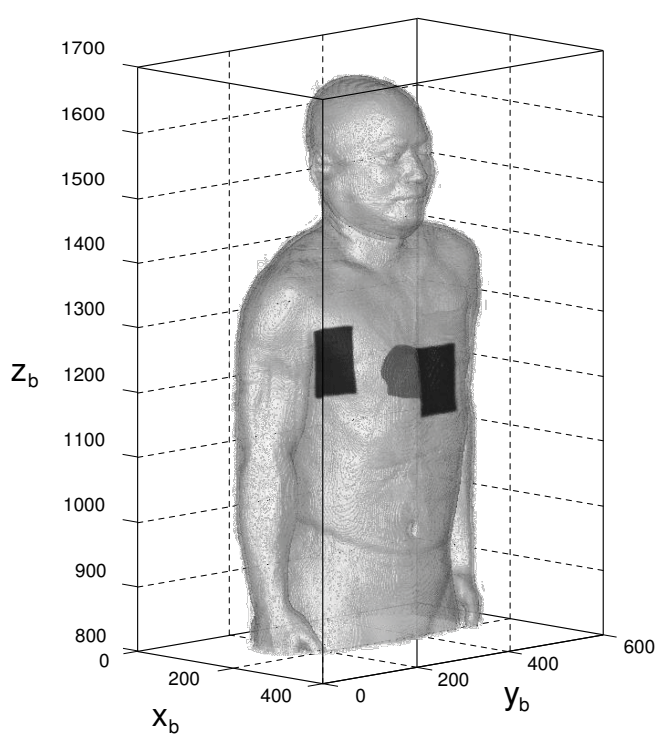

Fig. 10. Human torso with the heart and two defibrillator pads placed anteroposteriorly

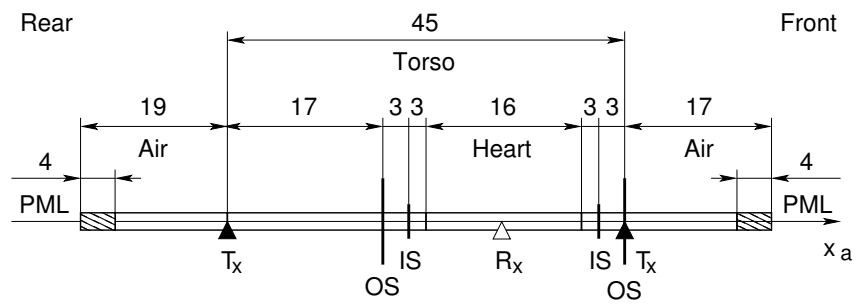

(a) Human torso with the defibrillator pads placed anteroposteriorly. Human torso, scenario setting in 1D as viewed by the main grid of the HSG. Observation point $R_{x}$ is in the middle of the heart. Rectangular pads $T_{x}$, where present, coincide with locations of the skin. Numerical values are given in the main grid units.

Rear

Front

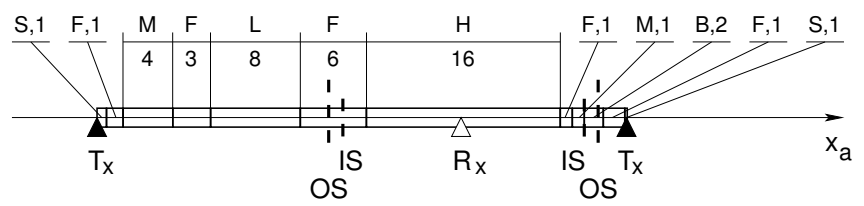

(b) Human body tissues between the defibrillator pads on the line crossing the heart at $x_{a} \in(5,51), y_{a}=42, z_{a}=244$ as viewed by the all coarse grid. Dotted lines denote the OS-IS locations in the main grid of the HSG. Following abbreviations are used: S-Skin, F-Fat, $\mathrm{M}-$ Muscle, L-Lung, $\mathrm{H}-\mathrm{Heart}$ and $\mathrm{B}-\mathrm{Bone}$. Numbers specify the tissue thickness in the main grid units.

Rear

Front

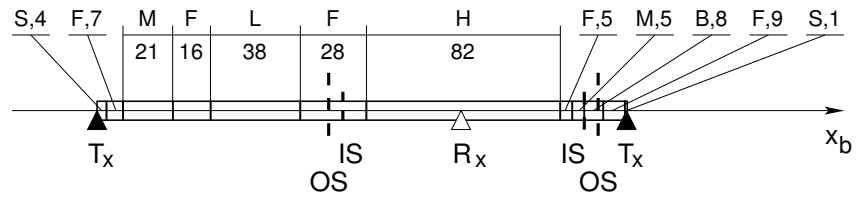

(c) Human body tissues between the defibrillator pads on the line crossing the heart at $x_{b} \in(26,251), y_{b}=211, z_{b}=1223$ as viewed by the all fine grid. Dotted lines denote the OS-IS locations in the subgrid of the HSG. Following abbreviations are used: S-Skin, F-Fat, M-Muscle, L-Lung, $\mathrm{H}-\mathrm{Heart}$ and $\mathrm{B}-$ Bone. Numbers specify the tissue thickness in the subgrid units.

Fig. 11. Human torso, scenario setting 


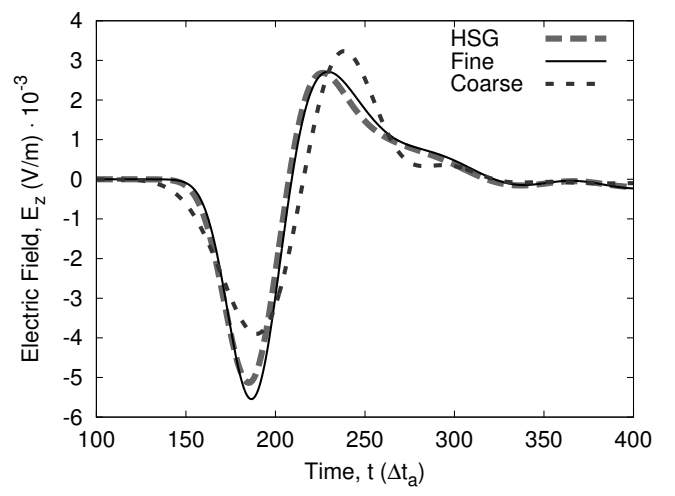

Fig. 12. Time domain observation at $R_{x}$ in the middle of the heart (See Fig. 11. The HSG signal matches the fine grid signal very well. No instability is visible during the entire simulation time of 1000 time steps.

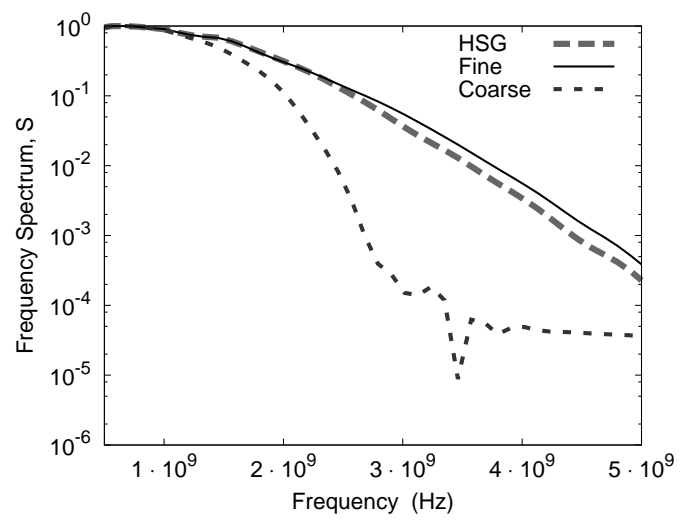

Fig. 13. Frequency domain observation at $R_{x}$ in the middle of the heart (See Fig. 11 .

frequency. However with the frequency dependent media this is only an approximation. A rigorous approach to take account of the Debye medium thinner than the cell size would be the generalisation of the formalisms in [17] and [18]. This will be the outcome of future work.

3) Observation: Up to $400 \Delta t_{a}$ of $E_{z}$ received at $R_{x}$ in the middle of the heart (See Fig. 11) is plotted in Fig. 12 A good matching between the HSG and the fine grid signals is observed. Fig. 13 shows $E_{z}$ in the frequency domain. The HSG signal preserved most of the high frequency components, which can be observed with the all fine grid simulation. On the other hand the all coarse grid has lost a significant amount of high frequency components during the propagation. This happened because the cut-off frequency of the coarse grid is five times lower than that of the fine grid.

\section{E. Error estimation}

The error $U$ between the HSG and the all fine grid signals was calculated in frequency domain as to

$$
U=\sqrt{\frac{\sum_{f}\left(S_{\text {Fine }}(f)-S_{\text {HSG }}(f)\right)^{2}}{\sum_{f}\left(S_{\text {Fine }}(f)\right)^{2}}},
$$

TABLE III

COMPUTATIONAL REQUIREMENTS OF HSG

\begin{tabular}{rrrrr}
\hline $\boldsymbol{p}$ & $\begin{array}{r}\text { Main grid } \\
\text { size }\end{array}$ & $\begin{array}{r}\text { Subgrid } \\
\text { size }\end{array}$ & $\begin{array}{r}\text { Memory } \\
{[\mathrm{MB}]}\end{array}$ & $\begin{array}{r}\text { CPU time } \\
{[\mathrm{s}]}\end{array}$ \\
\hline 0 & $80^{3}$ & 0 & 128 & 14 \\
1 & $80^{3}$ & $65^{3}$ & 142 & 108 \\
2 & $80^{3}$ & $90^{3}$ & 213 & 322 \\
3 & $80^{3}$ & $115^{3}$ & 322 & 911 \\
4 & $80^{3}$ & $140^{3}$ & 476 & 1713 \\
5 & $80^{3}$ & $165^{3}$ & 683 & 2849 \\
6 & $80^{3}$ & $190^{3}$ & 952 & 4806 \\
80 & 0 & $400^{3}$ & 6144 & 8835 \\
\hline
\end{tabular}

where $S_{\text {Fine }}$ and $S_{\mathrm{HSG}}$ signify the frequency spectrum values at the frequency $f$ obtained with the all fine grid and the HSG.

The minimum frequency in (11) was $0.5 \mathrm{GHz}$. When the maximum frequency is set to $6.0 \mathrm{GHz}$, the errors between the HSG and the all fine grid results were 1.32\% in Scenario 1, $11.40 \%$ in Scenario 2, 8.70\% in Scenario 3, 8.87\% in Scenario 4 and $2.63 \%$ in Human Torso Scenario. When the maximum frequency in (11) is set to $4.0 \mathrm{GHz}$, the errors were $0.38 \%$, $4.20 \%, 4.54 \%, 2.83 \%$ and $1.34 \%$, respectively.

\section{F. Computational requirements}

Computational requirements of the HSG were measured using an Intel Xeon computer with 8 CPUs (E5620 at $2.4 \mathrm{GHz}$ ) and 96 GB of memory running Scientific Linux 5.5. Here the main grid occupied $80^{3}$ voxels, while the IS box, the part of the subgrid within the IS, covered $(5 p)^{3}$ of the main grid domain. The integer $p$ is varied from 1 to 6 . The OS-IS separation was $3 \Delta s_{a}$. The PML was placed 5 cells away from the OS. In both the main and the subgrid domains the PML was 5 points thick. Thus, the effective main grid size equalled 703 The subgrid size was defined as $(5 p \times r+3 \times r \times 2+5 \times 2+5 \times 2)^{3}$.

The spatial and temporal steps were the same as in the basic accuracy tests (See Section IV-A). The CPU time was measured after running $100 \Delta t_{a}$, including the initialisation time. The computational requirements of the HSG are summarised in Table III] where the all coarse and the all fine grid cases are denoted as $p=0$ and $p=80$.

Fig. 14 is produced based on Table III Abscissa signifies the size of the IS box relative to the size of the main grid. The values on abscissa are defined as the ratio $\mathcal{R}=(5 p / 70)^{3}$. Ordinate shows the CPU time and the memory requirements of the HSG relative to the all fine grid (case $p=80$ in Table IIII).

Extrapolation applied to Fig. 14 suggests that the HSG with $r=5$ and the IS box of approximately $15 \%$ of the main grid size reaches the CPU time requirements of the all fine grid. The HSG requires the same amount of memory as the all fine grid in the case where the IS box covers $45 \%$ of the main grid. Thus, the HSG with $r=5$ outperforms the all fine grid case, when the IS box occupies less than $15 \%$ of the effective main grid domain.

\section{CONCLUSION AND FUTURE WORK}

This paper presented an extension of the HSG-FD-FDTD method from the one- to the three-dimensional case. Application of the one-pole Debye relaxation model together with 


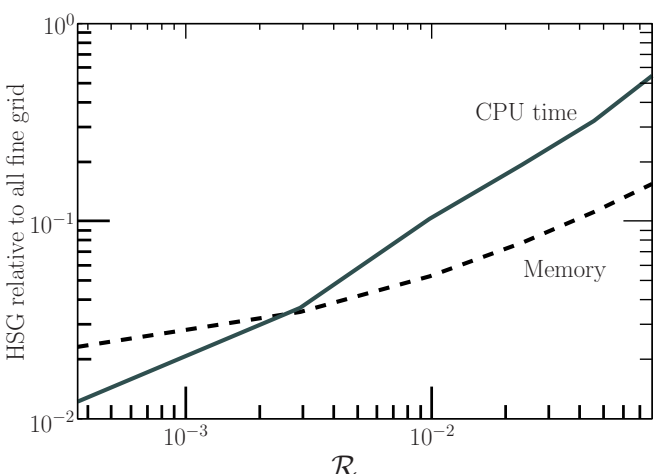

Fig. 14. CPU time and memory requirements of the HSG relative to the all fine grid

the HSG provided a versatile tool for electromagnetic wave simulation in dispersive materials.

The new 3D HSG-FD-FDTD method was extensively tested on various cases. The HSG method with a subgridding ratio of 5 showed very good matching with the all fine grid FD-FDTD method. No instability was visible in the dispersive HSG method during the $10^{6}$ simulation time steps. Finally, the HSG with $r=5$ outperforms the all fine grid case, when the IS box occupies less than $15 \%$ of the main grid volume.

Future work will consist of (i) the exploration of how permittivity $\varepsilon_{r}$ affects the general HSG stability, (ii) the adaptation of the Thin Slab method [17] for the correct calculation of media parameters when the main grid cells are thicker than a given medium and (iii) the application of the HSG method to calculate the current density and the flow distribution in the human heart.

\section{REFERENCES}

[1] R. Courant, K. Friedrichs, and H. Lewy, "On the partial difference equations of mathematical physics," IBM J., pp. 215-234, Mar. 1967.

[2] F. Costen, J.-P. Bérenger, and A. Brown, "Comparison of FDTD hard source with FDTD soft source and accuracy assessment in debye media," IEEE Trans. Antennas Propag., vol. 57, no. 7, pp. 2014-2022, 2009.

[3] "Revision of part 15 of the commission's rules regarding UltraWideband transmission systems," Federal Communications Commission, Washington, D.C. 20554, Tech. Rep. FCC 02-48, ET Docket 98 153, Apr. 22, 2002.

[4] M. W. Chevalier, R. J. Luebbers, and V. P. Cable, "FDTD local grid with material traverse," IEEE Trans. Antennas Propag., vol. 45, no. 3, pp. 411-421, 1997.

[5] B. Donderici and F. L. Teixeira, "Domain-overriding and digital filtering for 3-D FDTD subgridded simulations," IEEE Microw. Wireless Compon. Lett., vol. 16, no. 1, pp. 10-12, Jan. 2006.

[6] W. Pernice, F. Payne, and D. Gallagher, "Simulation of metallic nanostructures by using a hybrid FDTD-ADI subgridding method," in Intern. Conf. on Electromag. in Adv. Appl. IEEE, 2007, pp. 633-636.

[7] R. Kopecký and M. Persson, "Subgridding method for FDTD modeling in the inner ear," in Proc. of SPIE, vol. 5445, 2003, pp. 398-401.

[8] X. Xiaoli and W. Wenbing, "FDTD simulation of interstitial antenna for bone cancer microwave hyperthermic therapy," in IEEE Antennas Propag. Soc. Intern. Symp., vol. 2, 2004, pp. 1855-1858.

[9] A. Barchanski, M. Clemens, H. D. Gersem, T. Steiner, and T. Weiland, "Using domain decomposition techniques for the calculation of low-frequency electric current densities in high-resolution 3D human anatomy models," Intern. J. Comput. Mathematics in Electrical Electronic Eng., vol. 24, no. 2, pp. 458-467, 2005.

[10] J.-P. Bérenger, "A Huygens subgridding for the FDTD method," IEEE Trans. Antennas Propag., vol. 54, no. 12, pp. 3797-3804, Dec. 2006.

[11] _ "Extension of the FDTD Huygens subgridding algorithm to two dimensions," IEEE Trans. Antennas Propag., vol. 57, no. 12, pp. 3860 3867, Dec. 2009.
[12] - "The Huygens subgridding for the numerical solution of the Maxwell equations," J. Comput. Physics, vol. 230, pp. 5635-5659, 2011.

[13] F. Costen and J.-P. Bérenger, "Extension of the FDTD Huygens subgridding to frequency dependent media," Annals of Telecommunications, vol. 65 , no. 3 , pp. 211-217, 2010

[14] A. Taflove and S. C. Hagness, Computational Electrodynamics: The Finite-Difference Time-Domain Method, 3rd ed. 685 Canton Street, Norwood, MA 02062: Artech House, 2005.

[15] The RIKEN webpage, media parameters for the Debye relaxation model. Accessed: Oct. 3, 2011. [Online]. Available: http://cfd-duo. riken.jp/cbms-mp/

[16] A. J. Handley, Ed., Resuscitation Guidelines 2005. 5th Floor, Tavistock House North, Tavistock Square, London, WC1H 9JR: Resuscitation Council (UK), Dec. 2005, ISBN 1-903812-10-0.

[17] J.-P. Bérenger, "Plaques minces aux différences finies," in 6éme Colloque International et Exposition sur la Compatibilité Electromagnétique, CEM'92, Jun. 1992, pp. 298-303, (in French).

[18] B. Wei, S. Zhang, Y. Dong, and F. Wang, "A general FDTD algorithm handling thin dispersive layer," Progress In Electromagnetics Research, vol. 18, pp. 243-257, 2009.

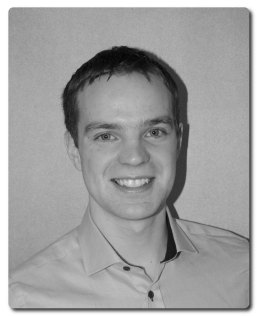

Maksims Abaļenkovs (S'08) was born in Riga, Latvia. He received his BSc (06) and MSc (07) degrees in Computer Science from the Heinrich-Heine-Universität in Düsseldorf, Germany and The University of Manchester in Manchester, UK. In 2011 Maksims Abaļenkovs has graduated from The University of Manchester with a $\mathrm{PhD}$ degree in Electrical and Electronic Engineering. Currently he is an engineer at EM Software \& Systems GmbH, Germany.

His research interests include the Finite-Difference Time-Domain methods, subgridding, dispersive materials modelling, Computational Electromagnetics, Bioelectromagnetics, computational methods in medicine, scientific computing and parallel programming languages.

Maksims Abalenkovs is a student member of the IEEE and SIAM.

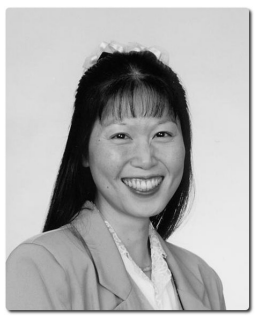

Fumie Costen (M'07) received the B.Sc. degree, the M.Sc. degree in electrical engineering and the Ph.D. degree in Informatics, all from Kyoto University, Japan. From 1993 to 1997 she was with Advanced Telecommunication Research International, Kyoto, where she was engaged in research on direction-of-arrival estimation based on Multiple SIgnal Classification (MUSIC) algorithm for 3-D laser microvision. She received an academic invitation at Kiruna Division, Swedish Institute of Space Physics, Sweden in 1996, and gained three patents from the research in 1999. From 1998 to 2000, she was with Manchester Computing in the University of Manchester, U.K., where she was engaged in research on metacomputing and has been a Lecturer since 2000. Her research interests include computational electromagnetics in such topics as a variety of the finite difference time domain methods for microwave frequency range and high spatial resolution and FDTD subgridding and boundary conditions. Her work extends to the hardware acceleration of the computation using general-purpose computating on graphics processing units, Streaming Single Instruction Multiple Data Extension ( SSE) and advanced vector extentions instructions. Dr. Costen received an ATR Excellence in Research Award in 1996 and a best paper award from 8th International Conference on High Performance Computing and Networking Europe in 2000. 


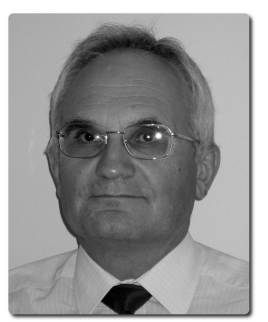

Jean-Pierre Bérenger (F'09) received the Masters degree in Physics from the University Joseph Fourier, Grenoble, France in 1973 and the Masters degree in Optical Engineering from the Institut d'Optique Graduate School, Paris, France in 1975. From 1975 to 1984 he was with the Département Etudes Théoriques, Centre d'Analyse de Défense, France, working on the propagation of waves and the coupling problems related to the nuclear electromagnetic pulse. He helped to popularise the Finite-Difference Time-Domain method in France. In 1984-1989 he was involved in the development of simulation software in the Département Nucléaire. From 1989 to 1998 he held a position as expert on the electromagnetic effects of nuclear disturbances. He is now a contract manager while staying active in the field of numerical electromagnetics, in such topics as low frequency propagation, absorbing boundary conditions, and the FDTD subgridding.

Dr Bérenger is a member of the Electromagnetics Academy and has been an Associate Editor of the IEEE TRANSACTIONS ON ANTENNAS AND PROPAGATION from 2006 to 2010

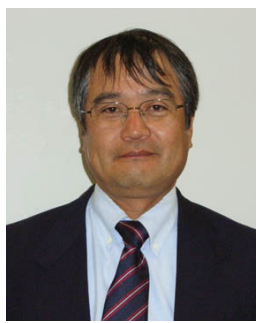

Ryutaro Himeno received his Doctor of Engineering degree from the University of Tokyo in 1988. In 1979, he joined Central Research Laboratories, Nissan Motor Co., Ltd., Yokosuka, Japan, where he has been engaged in the research of applying Computational Fluid Dynamics analysis to the car aerodynamic development. In 1998, he joined RIKEN (The Institute of Physical and Chemical Research) and is the director of Advanced Center for Computing and Communication and deputy program director of the Next Generation Computational Science Research Program at RIKEN. He is also a visiting professor at Hokkaido University, Kobe University and Tokyo Denki University. He currently studies curve balls in baseball games and blood flows of human bodies. He was a winner of Nikkei Science, Computer Visualization Contest in 2000 and Scientific Visualization Contest in 1996, and received JSME Computational Mechanics Division Award in 1997 and JSME Youth Engineer Award in 1988 He was also awarded Paper Award by NICOGRAPH in 1993, Giga FLOPS Award by CRAY Research Inc. in 1990 and other awards.

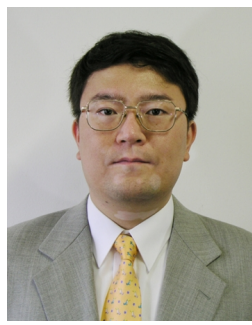

Hideo Yokota received his Doctor of Engineering degree from the University of Tokyo in 1999. In 1993, he joined Higuchi Ultimate Mechatronics Project, Kanagawa Academy of Science and Technology, Kawasaki, Japan. In 1999, he joined RIKEN (The Institute of Physical and Chemical Research) and is the contract researcher of Computational biomechanics unit. 2007-present Cell-scale Research and Development Team Leader,Research Program for Computational Science, RIKEN. 2010present Bio-research Infrastructure Construction Team Head, Advanced Science Institute, RIKEN. He is also a visiting Professor at Hokkaido University, Kobe University, Tokai University and the Tokyo University of Agriculture and Technology. He currently studies biomedical imaging and image processing to the biomedical simulation. Bioimaging Society, Best Image Award, 2005. The Commendation for Science and Technology by the Minister of Education, Culture, Sports, Science and Technology, The Young Scientists' Prize, 2008.

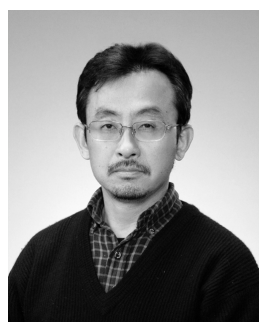

Masafumi Fujii received the B.E. and M.E. degrees in electrical and electronic engineering from Kobe University, Hyogo, Japan, in 1989 and 1991, respectively, and the Ph.D. degree in electrical and computer engineering from the University of Victoria, British Columbia, Canada, in 1999. He was with Sumitomo Metal Industries, Ltd., Japan, as a researcher from 1991 to 1998 , and a Humboldt Research Fellow in the Institute for High-Frequency Engineering at the University of Technology Munich, Germany, from 2001 to 2003 . He is currently an associate professor in the Department of Electric, Electronic and System Engineering at the University of Toyama, Japan. He was a visiting professor at the Institute of High-frequency and Quantum Electronics, University of Karlsruhe, Germany, in 2005. He co-authored Chapter 9 in "Computational electrodynamics - The finite-difference time-domain method", 3rd ed., Artech House, 2005. His research interest is in the field of numerical analysis of electromagnetic and optical fields in various nonlinear, dispersive, negative index, and random media. 